\title{
Chapter 4: Generating evidence in support of site planning and design
}

\section{The BlueHealth toolbox}

\author{
James Grellier, Himansu S. Mishra, Lewis R. Elliott, Susanne Wuijts \\ and Matthias F. W. Braubach
}

\section{Facilitating evidence-based planning and design of blue spaces}

In the first part of this book, we discussed in detail what we know about the impacts that blue spaces can have on human health and well-being and how changes made in those environments can potentially influence those effects. Faced with the design, repurposing or maintenance of urban blue spaces, decisions are routinely taken that potentially impact health, both positively and negatively. Choices made in other sectors - whether by planners, developers, industrialists, engineers, citizens or other decision-makers involved in the policy-making process - might also impact health and well-being through changes brought about in urban blue spaces.

In the case studies of evidence-based planning we were interested in implementing, testing and developing in the BlueHealth Project, we needed information on which to base the blue space planning and design decisions that would lead to harnessing the benefits to human health and well-being afforded by aquatic natural environments in the urban setting. The evidence-based approach to planning requires that various kinds of data be collected and analysed before and after any intervention is made. This provides the necessary understanding of the balance of risks and benefits associated with changes in an environment, in its usage, in the activities conducted in that space and in the health and well-being of its users and subsequently to plan in a way that maximises benefits and minimises risks. The evidence-based planning paradigm recognises that the prevention of a number of 21st-century public health challenges require that health and well-being be put centre stage in urban planning, creating high-quality environments that afford social interaction, physical activity and restorative recreation.

How do we understand blue spaces and the people in, on and around them in order to create an evidence base of sufficient breadth and depth to inform planning? An overriding aim of the BlueHealth project was to design an integrated set of tools to help facilitate this (Grellier et al. 2017). Part Two of this book provides detailed descriptions of the tools in this BlueHealth Toolbox (https://bluehealth2020.eu/resources/toolbox/), the rationale behind their development and examples of their application. While these tools cannot be used to understand entirely the complexity of an intervention in a blue space - particularly when using them to assess large-scale, multi-faceted interventions they do represent a consistent means of collecting information on key aspects of environments (including risks and benefits), the status and behaviours of their users and the opinions of all parties potentially affected by an intervention. Furthermore, these tools can be used to assess the changes brought about by intervention in blue spaces by re-applying the tools after intervention has taken place.

Improving our understanding of precisely how intentional alteration of urban blue spaces results in concomitant changes in the environment and human health requires the collection and analysis of various kinds of information. In order for this information to be considered evidence that serves to support a particular kind of intervention improving human health, it must be collected and analysed using robust tools and methods. Rigour in design, implementation 
and application of such tools is essential in ensuring that their findings are good representations of the situations found in the broad palette of urban blue spaces, are consistent and repeatable and are usable by the range of professionals and interested citizens who may need to apply them. The term 'evidence' need not be taken solely to mean quantitative information. Qualitative data are key to understanding people's relationships with their environment and the affordances within it, as well as the impacts that it has on them.

The tools that we developed can be envisaged primarily as a means of understanding the impacts on the environment and human health of any intervention made that affected the use of an urban blue space. However, relatively broad working definitions of the terms 'intervention' and 'site' were kept in mind as the tools were developed. A 'site' is a blue space, defined as an outdoor environment - either natural or manmade - that prominently features water and is accessible to humans either proximally (being in, on or near water) or distally/virtually (being able to see, hear or otherwise sense water) (Grellier et al. 2017). An 'intervention' is anything that has the potential to generate change in a blue space; this could be an alteration to its physical structure or fabric, environmental qualities, accessibility, maintenance, promotion, signposting or provision of information therein or social programmes that result in changes of use or perception of space. Any of these changes might result in change in use of the blue space, which might further impact - positively or negatively - the site's ecosystem or the health and well-being of those visiting it or living nearby. Change in use could also be actuated through the implementation of a policy, the introduction of new regulations or the enactment of new bylaws at local, regional, national or even supra-national levels.

A huge variety of interventions in the built and natural environment might be made that purposely provide people with opportunities to have increased contact with nature. We do not need to imagine such interventions only in the form of newly built structures or physical changes to a landscape but also as changes to the maintenance of an existing site, provision of information about a space or engagement with stakeholders. In BlueHealth, a set of community-level interventions - all aiming to improve health and well-being through altering use of a blue space were conducted that encompassed a variety of blue spaces, and it was their evaluation that provided the need for bespoke tools. These included so-called 'urban acupuncture' interventions (Lerner 2014), wherein relatively smallscale changes to the landscape were made at underused, inaccessible or negatively perceived sites, with the hypothesis that these changes might confer disproportionately large positive impacts on the use or enjoyment of those places by specific populations (see Chapter 15).

Importantly, even though we developed, tested and validated the tools using a testbed of community-level interventions that had improvement of human health as an ultimate goal, the use of the BlueHealth tools need not be constrained to changes made with such intentions. For example, the tools can be used to assess the effects of the construction of a gated community on a previously accessible coastline and on its local population and visitors. Indeed, from the point of view of applying the BlueHealth tools, an intervention could theoretically even be created by natural processes. Similarly, a section of footpath removed, or a beach swept away, by natural processes such as coastal erosion can be considered an 'intervention' from the point of view of using the BlueHealth tools in order to understand impacts on health and well-being of a population.

Understanding the scale of such impacts could be crucial in determining the value to society and the costs of engineering against similar events in future. Perhaps, somewhat counterintuitively, a lack of action on the part of humanity might even be considered an intervention. For example, where decision-makers have knowledge about potential co-benefits that might result from changing existing urban blue infrastructure but choose not to effect such a change, they have effectively 'intervened', and the impacts of this choice might be estimated indirectly through applying the tools.

The decision-making contexts in which interventions might be made vary considerably, and the objectives of such interventions are even more diverse. An urban site might be developed with the express intention of providing a blue space location for rest, recreation or physical activity. Alternatively, where water infrastructure projects such as storm 
drains are built to manage storm water or weirs are installed to protect against river flooding, and prevention of risks to human health is foremost in the minds of the decision-makers, the potential health benefits of recreational use of these spaces might additionally be considered a 'co-benefit'.

Additionally, the scale and geographic contexts of these projects can exhibit huge variation. With increases in scale and more urbanised settings, the range of stakeholders involved grows, further adding to the complexities of the governance process. Given these challenges, and since the breadth of situations in which interventions relating to blue spaces might be made is so huge, any tools developed to measure their impact on the health of humans and ecosystems are necessarily widely understandable, scalable and flexible.

Decisions relating to interventions are informed by baseline conditions at a site. However, there is considerable value in conducting an evaluation of the conditions before, during and after an intervention has been instigated and/ or completed. Both pre- and post-intervention application of the tools should be considered at several time points. In the case of physical changes to a site, environmental indicators may respond gradually to the intervention, dependent on the nature of those changes and the characteristics of the environmental system, but also be subject to seasonal or climate-dependent variation or human behavioural impacts. Similarly, indicators of impacts of an intervention on health would not generally be anticipated to materialise overnight, unless the health outcome was particularly common and the association with an environmental cause extremely strong (neither of which is typically the case).

Responses to an intervention may also be non-linear through time. For example, the novelty of a new public waterside park may result in greatly increased frequency of visits in the first weeks and months following its construction and then return to baseline levels again once this 'honeymoon period' is over. Alternatively, a stable, increased pattern of use might only be found after a period of 'burn in', perhaps as news of the park spreads through a population. Such changes do not have to be positive, of course, and could also represent decreases in ecological quality at a site, negative effects on neighbouring residents and so on. In short, while the tools described in the following chapters provide measures of the impact that interventions might make on a site and on individual and population health, consideration of the timing of their implementation is paramount to these measures being robust.

The tools have been developed for application at different scales, such as the site and its users, the local neighbourhood and its inhabitants, the city and its population and so on. In planning, their application allows the analysis of how a given intervention affects people and environments with different spatial relationships to the site itself. Additionally, as tools applied in research relating to health/well-being and interventions in built/natural environments, these tools have been used to investigate whether processes that operate at one spatial level are identifiable at others, thereby discerning potentially scalable aspects of a particular intervention.

It is entirely possible to use the tools described in the following chapters individually, at only a single scale. However, they have been carefully designed to work as an integrated toolbox, producing quantitative and qualitative information that can be analysed in parallel or in combination so as to better illuminate potential causal pathways between changes in the environment and human health. Additionally, some of these tools have been designed to elicit the same information at different geographical scales. The incorporation of questionnaire items, for example, in the tools aimed at populations at a given site, close to that site and at some distance to that site, allows for direct comparisons to be made regarding the use of - and the opinions relating to - a site, which may also be used to inform analyses of inequalities of opportunity, economic valuations and so on. Using a particular tool in isolation potentially leads to lost opportunities in terms of understanding the characteristics of a given blue space, the affordances therein and the associated potential for their benefitting the health and well-being of various populations. Similarly, key risks to health related to use of a given environment might not be identified if only one tool is used or if a tool is applied only at a single geographical scale.

From the perspective of the subject of this book, the most obvious application of the BlueHealth tools is in evaluating the impacts a particular planned intervention might make on the environment and/or on human health and 
well-being. This can be achieved through applying the tools before an intervention - that is, to form a clear picture of baseline conditions - and after an intervention. The change that has been mapped out between the two or more periods of pre-/post-intervention can - with appropriate caveats - be attributed to the changes effected on the ground. In this way, the tools provide information which supports planning and design in the urban landscape specifically geared towards provisioning of health and well-being benefits to a population, with minimal negative impact on the blue space environment.

However, the tools can be applied in other contexts, too, particularly in relation to the timing of the evaluative processes they facilitate. In this sense, it is entirely possible to apply them retrospectively where sufficient data exist elsewhere to inform prior baseline conditions. This might be conducted where a project is planned, is being built or has already been built, without consideration of the evidence-based approach or without any desire to specifically improve health and well-being by the original planners. In such a case, evidence could still be gathered - including retrospectively - using some of the BlueHealth tools, thereby allowing an evaluation of that project in terms of its impact on both the environment and human health. The tools might also be used to evaluate interventions made to achieve different goals. As already mentioned, although it might seem at odds with the overall emphasis of this book, the inter-sectoral applicability of these tools is one of their very important features.

It is apparent that not all tools can be used prospectively. For example, it is not possible to collect data on the patterns of user behaviour in an area of water yet to be reclaimed from the sea for construction of flood defences or a site that was previously private being made publicly accessible. Clearly, these are not limitations of the tool itself but rather constraints imposed by the realities of the sites.

Similarly, where an intervention has already taken place, it may not always be possible reliably to reconstruct information on environmental status or patterns of user behaviours retrospectively. In this case, however, it may be possible to impute information around the pre-intervention environmental status or human uses, provided that some data are either available, perhaps relating to a similar site nearby, or obtainable through interview with local experts or members of the local community. In such circumstances, it is still possible to apply the relevant tools; uncertainties and assumptions related to the information collected should then be detailed and reported.

Interventions and the tools used to evaluate them do not exist in a vacuum. Between identifying a planned intervention, selecting the method of evaluation and choosing the appropriate tools, through to collecting data and interpreting results, there is clearly an extensive interplay with various stakeholders with diverse interests in the planning process, the design of the intervention and in the site itself. The needs and wants of these stakeholders, their resources and their opinions may all shape the evaluation and use of the tools. They might feed into the interpretation of results and the design of any adaptation deemed necessary. It is entirely appropriate that these feedback loops should form a part of the planning, design and evaluative processes and be documented.

\section{Overview of the tools in the BlueHealth Toolbox}

The BlueHealth Toolbox comprises six tools that gather data on blue spaces in terms of their physical and ecological qualities (i.e. their environmental characteristics); the behaviours of people within them; and the perceptions, experiences and health and well-being status of the people using them or otherwise affected by them.

All of the tools can be used at any point in the process of changing a blue space site. They are most informative when used pre- and post-intervention, particularly where they are administered at multiple points in time within those phases.

While perhaps the application most relevant to the readers of this book is in purposely maximising the health and well-being benefits associated with a change made to a blue space with that goal in mind, the tools are also more generally applicable to the monitoring and evaluation of changes made to blue spaces with completely divergent goals. 


\section{BlueHealth Environmental Assessment Tool}

How can we characterise a given blue space, and what is its physical state? What are the qualities that set this site apart from other similar sites? What kind of flora and fauna does the water at the site support? Existing tools assessing outdoor environments in terms of the salutogenic (health-producing) potential have largely examined urban built environmental components, recreational environments, urban green spaces such as parks and urban design qualities related to certain activities (Mishra et al. 2020).

The BlueHealth Environmental Assessment Tool (BEAT) was developed to provide robust, objective measures of the environmental character of blue space sites, including both their terrestrial and aquatic systems. Divided into four steps, the tool guides the user through: (1) a preliminary desk-based study that collects data on a site's location and character; (2) an onsite evaluation of the site's character - a first impression; (3) the main survey, in which aspects of social, aesthetic and physical characteristics of the site are scored; and (4) an evaluation of the aquatic ecosystem.

Two somewhat distinct versions of the tool have been developed. A 'Professional BEAT' is intended for professional users, such as landscape architects, ecologists, recreation planners, urban planners or hydrologists who have expert knowledge of the relevant domains and may have sophisticated methods or instruments for assessing many of the factors. Although the BEAT only requires two assessors, a professional application of the tool might involve a team of experts who can independently collect, moderate and interpret the data; relate data collected on site to existing monitoring data (for example, air or surface water quality); and analyse the factors with one another rather than treating them separately. A 'Community BEAT', on the other hand, is aimed at individuals and community groups with an interest in their local environment that are seeking information on aspects of a blue space, perhaps as a citizen science or educational project or in support of funding applications for other activities. This version of the tool is shorter and less complicated than the professional version.

The BEAT is primarily designed to be used as an online tool, but it is also available for download, which allows for a printed version to be used on sites where assessors are not connected to the internet. There are several opportunities for using BEAT in tandem with the decision support tool (DST). Whereas the BEAT serves chiefly to identify characteristics of the site - some of which may indeed be hazards or potential sources of health benefit - the DST serves to describe risks and benefits to health, contextualise them and provide guidance on design features that might reduce the impacts of health risks and enhance and maximise possible benefits. DST users may benefit from prior application of the BEAT to generate a solid understanding of the environmental character of the blue space, which can then be used as a baseline for identifying potential risks and benefits in the DST. Chapter 5 explains the design and use of the BEAT in detail.

\section{BlueHealth Behavioural Assessment Tool}

What do people do at blue spaces? If they are active, what kinds of physical activity are they engaged in, and how much of it are they doing? If they are passive, what is the nature of their passive behaviour? How are their activities in a space associated with characteristics of the space and with aspects of the site status at different points in time? To answer these questions, the BlueHealth Behavioural Assessment Tool (BBAT) was developed.

The starting point for this work was the System for Observing Play and Recreation in Communities (SOPARC) (McKenzie et al. 2006). Whereas SOPARC serves to evaluate the amount of different kinds of physical activity in recreational settings, the BBAT has been designed to systematically capture all kinds of human behaviours and interactions at a site, as well as the weather and water conditions (e.g. tide, waves, etc.) at the time of observation. It captures who is doing what and where and allows researchers to make comparisons between different groups and activities. The BBAT outputs can be presented spatially in geographic point, heat map or hot-spot analysis forms, providing a visual way to assess and discuss results. Interrogation of these outputs allows the identification of affordances in blue spaces and how these relate to specific user groups. 
The BBAT is a site-level tool. Background data collection and initial site mapping are comparable and parallel processes to those collected in the application of the BEAT. The outputs may well also prompt decision-makers to identify particular issues using the DST, depending on where the site users congregate and the types of activities they are observed doing. Chapter 6 explains how the BBAT was developed and how it can be used and also compares it with the SOPARC method.

\section{Public Participation Geographic Information System: collaborative mapping of affordances}

A blue space located at a particular place in a city might afford or be available for interactions for people living some distance away. Public Participation Geographic Information System (PPGIS; sometimes known, since it collects "soft values", as SoftGIS) is a geographical information system methodology that enables participatory mapping by allowing residents of a city, for example, to share their knowledge about their living environment with urban planners and researchers (Kahila and Kyttä 2009). In the context of BlueHealth, the commercially available Maptionnaire system (https://maptionnaire.com/) was used to understand how local residents used the blue spaces in the cities under study and to gauge what these places meant to them. For example, the system can be used to identify (and to begin to understand why) the favourite blue spaces in a city, those that are avoided, blue spaces that are under- or overused and opportunities for developing new blue spaces in areas where no natural ones are present.

One limitation of all pre-post intervention work is knowing whether changes observed in behaviours (e.g. visits) are genuinely related to an intervention at a site or in fact reflect general changes in attitudes and behaviours over time unrelated to that intervention. Enabling local residents to comment on their experiences in local blue spaces provides a more complete picture of the importance and relevance of changes made at these sites. The application and illustration of the PPGIS is covered in Chapter 7.

\section{BlueHealth International Survey}

The BlueHealth International Survey (BIS) is a bespoke online survey (in the form of a questionnaire) designed to answer the same questions as the BlueHealth Community Level Survey (BCLS; see subsequently) but at a higher spatial scale. The BIS has so far been administered to nationally representative online panels (i.e. to preregistered participants of online surveys) in some 18 countries, mainly in Europe but also in North America, Australia and Hong Kong. It can also be used at the city level by targeting its administration to online panels resident in particular cities.

Used in isolation, the BIS allows the collection and analysis of extremely rich data on how frequently different kinds of blue spaces are visited, how far people travel to reach them and on internationally validated self-reported measures of health and well-being. Cross-sectional analyses provide insights into, for example, the links between health and well-being and the frequencies of visits to blue spaces.

Used as a component of the BlueHealth Toolbox, however, in parallel with other tools, it provides a useful national or regional benchmark of visit frequencies and self-reported health status with which data collected at a city or site level can be compared. Where an intervention is found to work - in the sense that it has net beneficial effects on health of a local population, as measured using the BCLS or BBAT, for example - data from the BIS may prove useful in gauging how representative characteristics and behaviours of that population are of a national population. This information would, for example, potentially lend support to those making the case for scaling up the intervention for application elsewhere in the country. A detailed description of the BIS is provided alongside the BCLS in Chapter 8.

\section{BlueHealth Community Level Survey}

How frequently do people visit blue spaces? How do they reach them? What activities do they do there? How do their visits make them feel? How does the state of the environment affect their propensity to visit natural spaces featuring water? 
The BlueHealth Community Level Survey is a questionnaire designed to answer these questions. It was designed to serve as a shorter, site-specific version of the BlueHealth International Survey (see previously); the BCLS is particularly suited for administration to communities affected by changes made to a local site. The inclusion of items common to the BIS allows for contextualisation and comparison of findings made locally with those made at a national or city level. Where engagement with people living further afield is desirable, we recommend the use of the PPGIS instead of the BCLS.A full description of how the BCLS was developed and how it can be implemented is presented in Chapter 8.

\section{BlueHealth Decision Support Tool}

The BlueHealth Decision Support Tool guides a user through a series of steps to identify the key risks and benefits to health and environment of a given blue space setting. It provides urban planners, architects, policy makers and estate managers with a novel means of approaching the planning, management and maintenance of blue infrastructure, with both health promotion and the management of potential health and environmental risks in mind. It highlights threats and ways to mitigate them, as well as spotting possible opportunities for boosting public health and well-being.

The tool includes information for different blue settings such as marine environments, rivers, lakes, urban water bodies, blue spaces in green settings (parks, etc.) and ornamental blue spaces (e.g. fountains). It features guidance for planning and management on three key elements: (1) health risks related to blue spaces, such as drowning, illnesses, vector-borne diseases, or climate-specific factors (e.g. UV exposure); (2) human health and well-being benefits, such as opportunities for physical activity or improvements in mental and social well-being; and (3) improved ecosystem and environmental benefits, such as improved air and water quality, habitats and biodiversity. Each outcome is described in terms of its relevance to health, identifying the scale of the threat or opportunity and the human populations most likely to be affected.

The DST is an online tool developed to be used at a site, or at least by evaluators with a very good knowledge of the characteristics of a site. The development and application of the DST are described in Chapter 9.

\section{Using the tools together: the BlueHealth Toolbox as an integrated assessment framework}

Individually, each tool provides information on a key set of characteristics relating to the site, typically before and after an intervention. Used in combination within a single integrated assessment framework (the BlueHealth Toolbox), these tools have the potential to provide an evaluation team with a very rich dataset on multiple aspects of a blue space and the human populations affected by it before, during and after interventions. It has only been through carefully ensuring that the tools use internally consistent definitions, concepts and questionnaire items that such integration is possible.

All sites have environmental characteristics and qualities that can be evaluated. Similarly, urban blue spaces are all subject to some kind of use, which, alongside behaviours of humans interacting within those spaces, can be assessed. Individuals have a measurable health and well-being status. Collecting data on each of these domains can be done using tools available in the BlueHealth Toolbox, but by no means should a practitioner consider that all tools must be applied to all sites or at multiple points in time.

There are at least three issues, or dimensions, to consider when selecting which tools to employ in assessing a specific intervention and how to employ them. These dimensions relate to: the spatial scale(s) at which the effects of an intervention are considered most relevant, the time scale(s) over which that evaluation should be conducted and the population(s) for whom health and well-being impacts are considered.

Some tools are quite clearly designed to assess characteristics at the site level, such as the BBAT. The evaluator applying the tool can be confident in applying this only at the site, as it is designed specifically to understand use of 
the blue space itself. Other tools can be used at multiple spatial levels (see Figure 4.1). For example, although it was designed to capture information at the national level, there is no reason the BIS cannot be administered at international, regional and city levels. The only constraint here is whether suitable online panels exist at those levels, that is, whether sufficient numbers of responses would be obtainable. The flexibility of the means of administering the BCLS, which can be printed on paper and posted, delivered as an online survey or used in face-to-face interviews, means that it might be used on- and off-site and with specific populations. BCLS interviewers could approach users of a blue space in the space itself or administer the survey to nearby residents at their doorsteps, for example. The important aspect of both is that they share common questionnaire items, so analyses at different spatial levels are automatically comparable.

The time points at which different tools should be evaluated needs careful consideration (Figure 4.2). Also, there is little value in assessing environmental characteristics of a site multiple times while only evaluating health status once. The combination of these two datasets at the same multiple time points generates far more opportunities for understanding the impacts of an intervention. The characteristics of most environments vary over time, diurnally, seasonally, annually and so on, and so do their usage and the health status of those using them. It is essential that the elucidation of the true impacts of the intervention on health not be obscured by patterns in the data observed

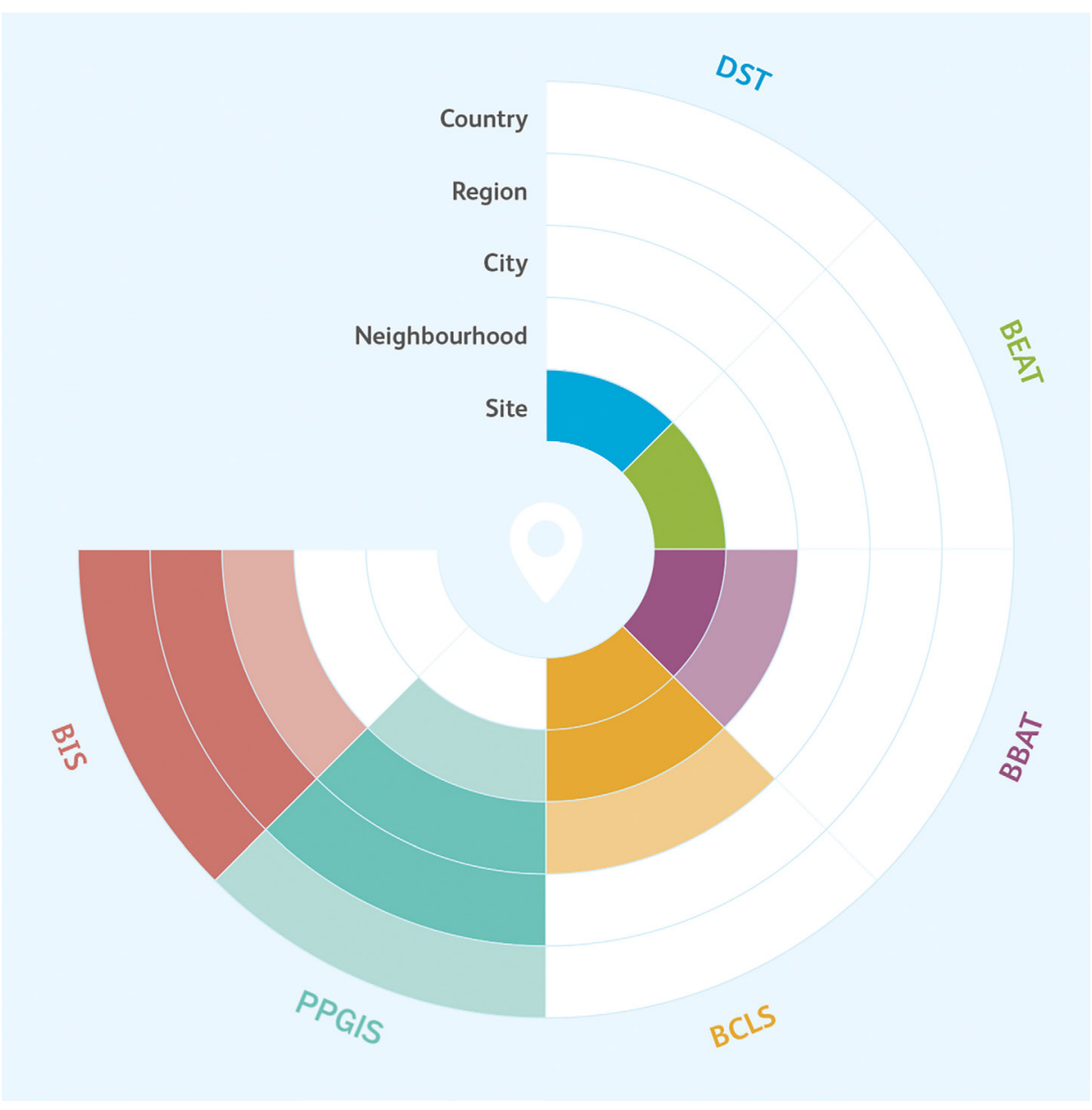

Figure 4.1

Spatial scales at which the tools in the BlueHealth Toolbox are designed to be employed 


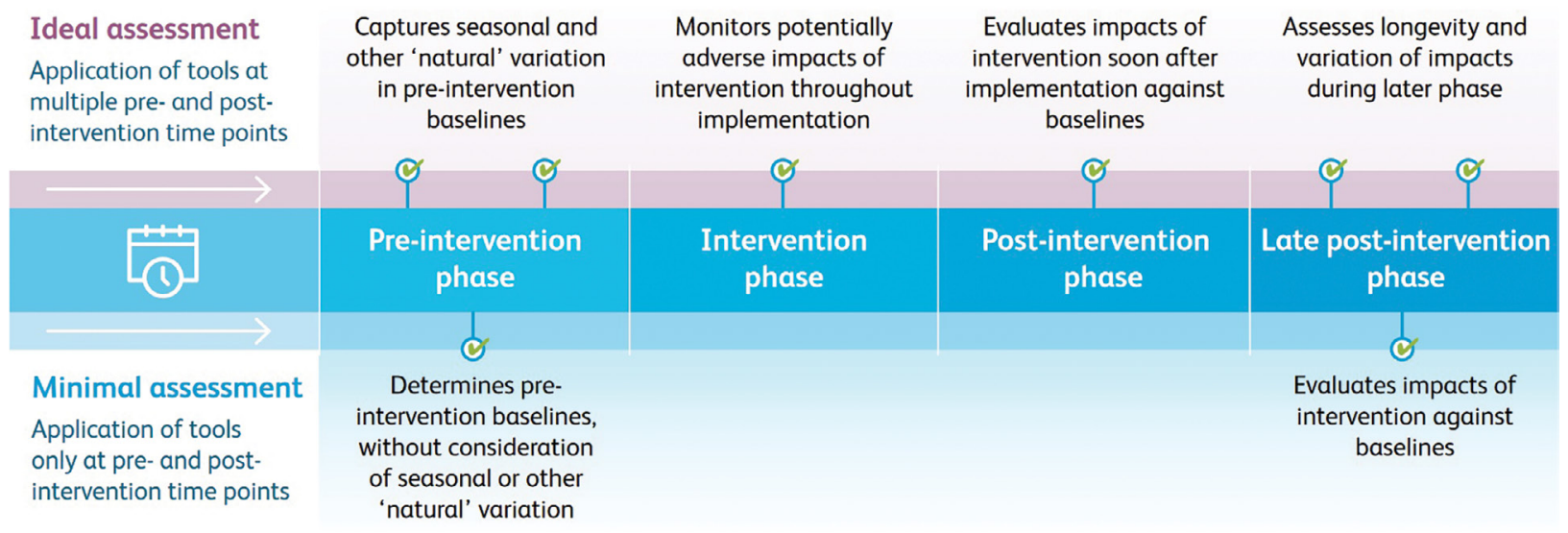

Figure 4.2

Temporal aspects of assessing the impacts of an intervention using the BlueHealth Toolbox

only due to lack of consideration of change affected by factors potentially external to the analysis of interest such as seasonality, for example.

Consideration should be given to assessing several populations when investigating how people use, feel about or are otherwise affected by a site and changes in it. On-site interviews will only ever provide information about current users of an urban blue space and nothing about those who are prevented from visiting by any of several barriers. Doorstep interviews and the like provide useful information about those populations assumed to have a relationship to the site (even if they do not visit it), but some blue spaces are potential destinations for populations spread across whole cities. PPGIS provides a partial solution to this, but it too relies on very partial and potentially unrepresentative sampling as a result of it being an online platform.

The teams that are brought together to work on evaluating an intervention at a given site using the BlueHealth Toolbox can comprise researchers and practitioners representing a wide range of disciplines, and they may also include or be led by stakeholders who have no professional experience in such evaluations. As in any interdisciplinary work, it is essential that methods and results of all the tools be understood sufficiently by all those working on the assessment that they are able to have informed discussions about the overall evaluation of a site.

The BlueHealth Toolbox is a collection of what might be described as 'diagnostic tools'. They can be used to collect a variety of information on the places and the people using or otherwise affected by them; they are not specifically designed to understand the governance processes that facilitate the realisation and management of blue spaces or the city in which they are located. The processes by which such knowledge is obtained are determined by, for example, the participating stakeholders' local ambitions, the instruments and means available and an understanding of the water system and its interactions with the local environment. Because of the complexities of these processes, no single, highly prescriptive method or tool aimed at building such knowledge is appropriate. Numerous existing methodologies are, however, available to analyse governance and to identify gaps and opportunities Wuijts et al. 2020).

Within BlueHealth, stakeholders have been involved in different ways in the implementation and evaluation of all interventions. The specific participatory approaches used to develop long-term planning of BlueHealth interventions are discussed at length in Chapter 16. 
James Grellier et al.

References

Grellier, J., White, M.P., Albin, M., Bell, S., Elliott, L.R., Gascon, M., Gualdi, S., et al. (2017). BlueHealth: A Study Programme Protocol for Mapping and Quantifying the Potential Benefits to Public Health and Well-Being from Europe's Blue Spaces. BMJ Open 7(e016188): 1-10.

Kahila, M., and Kyttä, M. (2009). SoftGIS as a Bridge-Builder in Collaborative Urban Planning. In Planning Support Systems Best Practice and New Methods, edited by Geertman, S. and Stillwell, J.C.H., 389-411. Dordrecht: Springer Netherlands.

Lerner, J. (2014). Urban Acupuncture. Washington, DC: Island Press.

McKenzie, T.L., Cohen, D., Sehgal, A., Williamson, S., and Golinelli, D. (2006). System for Observing Play and Recreation in Communities (SOPARC): Reliability and Feasibility Measures. Journal of Physical Acticity \& Health 3(s1): S208-222.

Mishra, H.S., Bell, S., Vassiljev, P., Kuhlmann, F., Niin, G., and Grellier, J. (2020). The Development of a Tool for Assessing the Environmental Qualities of Urban Blue Spaces. Urban Forestry \& Urban Greening 49(January): 126575.

Wuijts, S., Friederichs, L., Hin, J.A., Schets, F.M., van Rijswick, H.F.M.W., and Driessen, P.P.J. (2020). Governance Conditions to Overcome the Challenges of Realizing Safe Urban Bathing Water Sites. International Journal of Water Resources Devlelopment 00(00): 1-25. 